\title{
Perturbative analysis of an n-Ising model on a random surface
}

\author{
Shinobu Hikami ${ }^{1}$ and Edouard Brézin ${ }^{2}$ \\ ${ }^{1}$ Department of Pure and Applied Sciences, University of Tokyo, Meguro-ku, \\ Komaba, 3-8-1, Tokyo 153, e-mail address:a87353@tansei.cc.u-Tokyo.ac.jp \\ ${ }^{2}$ Laboratoire de Physique Théorique de l'Ecole Normale Supérieure,24 rue \\ Lhomond,F-75231 Paris Cedex 05, e-mail address:brezin@physique.ens.fr ${ }^{\star}$
}

\begin{abstract}
Two dimensional quantum gravity coupled to a conformally invariant matter field of central charge $c=n / 2$, is represented, in a discretized version, by $n$ independent Ising spins per cell of the triangulations of a random surface. The matrix integral representation of this model leads to a diagrammatic expansion in powers of the cosmological constant for fixed genus. From the behaviour of this expansion at large orders, when the Ising coupling constant is tuned to criticality, one extracts the values of the string susceptibility exponent. We extend our previous calculation to order eight for genus zero and investigate now also the genus one case in order to check the possibility of having a well-defined double scaling limit even for $c>1$.
\end{abstract} September 1992

\footnotetext{
* Unité prope du Centre National de la Recherche Scientifique,associée à l'Ecole Normale Supérieure et à l'Université Paris-Sud.
} 
A discretized approach to two-dimensional quantum gravity coupled to matter fields of central charge $c=n / 2$, is easily formulated as an integral over $2^{n} N \times N$ Hermitian matrices ${ }^{[1]}$. In this model each cell of a discretized random surface carries $n$ Ising spins. For a fixed triangulation these $n$ species do not interact and the matter partition function is simply the $n$-th power of one Ising spin; however the various species are effectively coupled by the sum over the triangulations. In a previous article ${ }^{[2]}$, we found that at relatively small order in perturbation theory in the cosmological constant, we recovered with a surprizing accuracy, the known results for the string susceptibility exponent $\gamma_{\text {string }}$, for $c<1$ and planar surfaces. For larger values of $c$, at least up to $c=4$, we did not see any sign of the tachyonic instability of the theory. Similar conclusions on the absence of instabilities were obtained through Monte-Carlo simulations of the same model ${ }^{[3]}$, but the values of the exponents were not reported. There were several attempts to evaluate the string susceptibility exponent for $c>1$, by generating random surfaces embedded in a space of more than one dimension, but the results have not really converged so $\operatorname{far}^{[4]}$.

In our previous work ${ }^{[5]}$, we had expanded the free energy up to sixth order in the cosmological constant $g$, for surfaces of genus zero. In this letter we extend the series to order eight, and include surfaces of genus one, in order to check the variation of the string susceptibility with genus which governs the existence of a double scaling limit. The expansion in powers of $g$ is given by $\phi^{4}$ Feynman diagrams in which each vertex carries $n$ spins, i.e. $2^{n}$ "flavors". The Ising interaction $\beta$ is

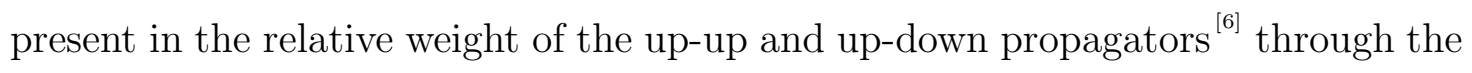
parameter $a$ defined as

$$
a=\exp (-2 \beta)
$$

The sum over the $n$-Ising species is, for a given diagram, the $n$-th power of a one Ising sum, which is a polynomial in the variable $a^{2}$. When the parameter $a$ 
varies and reaches its critical value, for instance $1 / 4$ for one Ising spin, the theory crosses over from pure gravity, with $\gamma_{\text {string }}=-1 / 2$ to a coupling with conformally invariant matter with central charge $n / 2$ and an exponent $\gamma_{\text {string, }}$, which is for one Ising spin, but that we have tried to determine for $n$ spins.

The vertex of the $\phi^{4}$ hermitian matrix model, carries four different lines of different colors $i, i=1, \ldots, N$. At $l$-th order the permutations of the $4 l$ color indices, generate the various diagrams, and this requires a fast algorithm. If we use unitary instead of hermitian matrices, we obtain the same type of diagrams, with two incoming and two outgoing lines at each vertex, and the diagrams differ only by symmetry factors. In fact these symmetry factors are the same for the two ensembles at genus zero, but there are differences at genus one. With this unitary ensemble we generate the Feynman diagrams with the same tree sorting algorithm that was used for the study of the perturbation theory at large order of the LandauGinzburg Hamiltonian ${ }^{[7]}$. In this method, the symmetry factor is automatically taken acount by the generation of all possible line combinations. Disconnected diagrams are avoided by a computation of the determinant of the adjacency matrix; it is made of 2 on the diagonal, and -1 for the elements corresponding to vertices connected by a line and some of the -1 of the element is replaced by 0 . This determinant is equal to the number of Euler trails for a connected graph, but it vanishes for a disconnected diagram. ${ }^{[8]}$

We have a certain number of checks on our eighth order result: the one Ising spin first ${ }^{[9]}$. Then, if either $a$ or $n$ vanish we must recover the series of pure gravity $^{[10]}$. The free energy is given by

$$
\frac{F}{2^{n}}=\sum_{k} C_{k} g^{k}
$$

and the coefficient $C_{k}$ behaves for the large order as

$$
C_{k} \simeq A^{k} k^{-3+\gamma_{s t r i n g}}
$$

The ratio $f_{k}=C_{k} / C_{k-1}$ gives the value of the string susceptibility exponent by the 
extrapolation, for example we have used the following ratio method in the previous analysis. $^{[11]}$

$$
\begin{gathered}
A_{k}=(1+k) f_{k}-k f_{k-1} \\
\gamma_{s t r i n g}=3-k(1+k)\left(f_{k}-f_{k-1}\right) / A_{k}
\end{gathered}
$$

Another method of the analysis, which we employed before, is the Padé approximation for the ratio $f_{k}$ by

$$
f_{k}=A\left(1-\frac{1}{k}\right) \frac{\left(1+\frac{b_{1}}{k}\right)}{\left(1+\frac{c_{1}}{k}\right)}
$$

from which the string susceptibility $\gamma_{s t r i n g}=2+b_{1}-c_{1}$ is estimated. The unknown values $A, b_{1}$ and $c_{1}$ are determined from $f_{k}, f_{k-1}$ and $f_{k-2}$ for the fixed order $k$. This Padé becomes exact in the pure gravity $a=0$ case with the value $b_{1}=-1 / 2$ and $c_{1}=2$. This analysis is more convergent and it is convenient to observe the singularity due to the finite order perturbation, especially for the separated peaks at the large value of $a$. This method however fails to obtain the correct result for $n=2(c=1)$ since (6) has no $\log k$ correction. For $n=2$, we have checked that our eighth order perturbation agrees with $C_{k} \simeq A^{k} k^{-3} / \log k$ behavior, for $a=1 / 4$. So indeed we have $\gamma_{\text {string }}=0$ for $n=2$, although we could not determine with precision the critical value $1 / 4$ of a. The $1 / \operatorname{logk}$ in $C_{k}$ is indeed compatible with the logarithmic deviation to KPZ scaling found in the $\mathrm{c}=1$ problem. ${ }^{[12]}$

In Fig.1a-1c, the string susceptibility $\gamma_{\text {string }}=2+b_{1}-c_{1}$ in (6) is plotted for $\mathrm{n}=1, \mathrm{n}=4$, and $\mathrm{n}=6$ respectively. At $a=0$ and $a=1$, the string susceptibility exponent becomes exactly $\gamma_{\text {string }}=-1 / 2$. And it takes the crossover from the pure gravity fixed point to the new fixed point at the critical vaue of $a$. In Fig.1a-1c, the lines of a,b,c and d correspond to the Padé approximaion (6) for the string susceptibility exponent of order $g^{5}, g^{6}, g^{7}$ and $g^{8}$ respectively. In Fig 1a $(\mathrm{n}=1)$, we 
find that at the critical point $a=1 / 4, \gamma_{\text {string }}=-0.34$, which is very close to the exact value of $-1 / 3$. The critical value of a is known from the exact solution. ${ }^{[13]}$ For $n=3$, we have a slightly negative peak value of $\gamma_{\text {string }}$ by this method. There is a logarithmic singularity for $n=2$, this may still influence the $n=3$ series, even if there is no $\log$ singularity in that case. The ratio metod of (5) for $n=3$ case gives $\gamma_{\text {string }} \simeq 0.02$. For $n=4, \gamma_{\text {string }}$ becomes 0.04 as shown in Fig $1 \mathrm{~b}$. The $\mathrm{n}=2$ logarithmic singularity may still lead to underestimating the exponent for $n=4$. In the case $n=6$ (Fig.1c), a second peak appears for larger values of a, but its height decreases at higher orders, and this second peak seems to merge with the first one near $a=0.2$. We estimate the string susceptibility $\gamma_{\text {string }}$ as 0.14 for $n=6$. The broken line is a guide line and the crossing point is the extrapolated value. In $n=8$, the second peak becomes more pronounced but it also approaches to the first small peak around $a=0.15$. In this case, assuming the two peaks merge at the same point, we estimate $\gamma_{\text {string }} \simeq 0.25$. The obtained values up to $n=8(c=4)$ are not far from $(c-1) / 12$, which is the real part of the KPZ formula, ${ }^{[14]}$ valid for $c<1$,

$$
\gamma_{\text {string }}=\frac{c-1}{12}-\frac{\sqrt{(c-1)(c-25)}}{12}
$$

although our values are lower.

The free energy of the genus one is evaluated from the diagram of the genus zero by permutation of the four lines at the vertices. We calculate the ratio of each order of genus for a fixed diagram, and then normalize the coefficients of each diagram by the genus zero results. The genus one case for the pure gravity has been studied ${ }^{[15]}$, from which we checked our perturbation series. The large order behavior of the coefficient $C_{k}$ of the genus one of the free energy is also written by

$$
C_{k} \simeq A^{k} k^{-3+\tilde{\gamma}_{s t r i n g}}
$$

We have a scaling relation of $\tilde{\gamma}_{s t r i n g}$ for $h$-genus case, 


$$
\tilde{\gamma}_{\text {string }}=\gamma_{\text {string }}+h \gamma_{\text {string }}^{\prime}
$$

For the genus one, we have $h=1$,

$$
\gamma_{\text {string }}+\gamma_{\text {string }}^{\prime}=2
$$

if $c<1^{[16]}$. This relation may be violated for $c>1$ and we are unable to assume this relation. The free energy of the genus one for the Hermitian matrix model is expanded up to order six, and here we present the result up to fifth order result, 


$$
\begin{aligned}
& E_{1}(g)=-g+g^{2}\left[20\left(1+a^{2}\right)^{n}+10\left(1+a^{4}\right)^{n}\right] \\
& -g^{3}\left[256\left(1+a^{2}\right)^{2 n}+416\left(1+a^{2}+2 a^{4}\right)^{n}+\frac{608}{3}\left(1+3 a^{2}\right)^{n}+\frac{544}{3}\left(1+3 a^{4}\right)^{n}\right] \\
& +g^{4}\left[2816\left(1+a^{2}\right)^{3 n}+6400\left(1+4 a^{2}+3 a^{4}\right)^{n}+2080\left(1+6 a^{2}+a^{4}\right)^{n}\right. \\
& +896\left(1+a^{2}+4 a^{4}+a^{6}+a^{8}\right)^{n}+880\left(1+6 a^{4}+a^{8}\right)^{n} \\
& +9856\left(1+a^{2}+5 a^{4}+a^{6}\right)^{n}+6144\left(1+2 a^{2}+5 a^{4}\right)^{n}+4480\left(1+3 a^{2}+3 a^{4}+a^{6}\right)^{n} \\
& \left.+3712\left(1+2 a^{2}+3 a^{4}+2 a^{6}\right)^{n}+2912\left(1+5 a^{4}+2 a^{6}\right)^{n}\right] \\
& -g^{5}\left[28672\left(1+a^{2}\right)^{4 n}+63488\left(\left(1+a^{2}\right)\left(1+4 a^{2}+3 a^{4}\right)\right)^{n}\right. \\
& +32768\left(\left(1+a^{2}\right)\left(1+2 a^{2}+3 a^{4}+2 a^{6}\right)\right)^{n}+78848\left(\left(1+a^{2}\right)\left(1+6 a^{2}+a^{4}\right)\right)^{n} \\
& +77824\left(\left(1+a^{2}\right)\left(1+3 a^{2}+3 a^{4}+a^{6}\right)\right)^{n} \\
& +107520\left(\left(1+a^{2}\right)\left(1+2 a^{2}+5 a^{4}\right)\right)^{n} \\
& +81920\left(\left(1+a^{2}\right)\left(1+a^{2}+5 a^{4}+a^{6}\right)\right)^{n}+34816\left(1+3 a^{2}\right)^{2 n} \\
& +34304\left(\left(1+3 a^{2}\right)\left(1+a^{2}+2 a^{4}\right)\right)^{n}+21606.4\left(1+10 a^{2}+5 a^{4}\right)^{n} \\
& +49664\left(1+6 a^{2}+5 a^{4}+4 a^{6}\right)^{n}+125952\left(1+4 a^{2}+9 a^{4}+2 a^{6}\right)^{n} \\
& +92672\left(1+3 a^{2}+7 a^{4}+5 a^{6}\right)^{n}+47616\left(1+2 a^{2}+7 a^{4}+4 a^{6}+2 a^{8}\right)^{n} \\
& +18944\left(1+3 a^{2}+6 a^{4}+3 a^{6}+3 a^{8}\right)^{n}+176128\left(1+2 a^{2}+9 a^{4}+4 a^{6}\right)^{n} \\
& +60928\left(1+a^{2}+9 a^{4}+3 a^{6}+2 a^{8}\right)^{n} \\
& +38912\left(1+3 a^{2}+11 a^{4}+a^{6}\right)^{n}+97792\left(1+a^{2}+7 a^{4}+7 a^{6}\right)^{n} \\
& +44032\left(1+2 a^{2}+10 a^{4}+2 a^{6}+a^{8}\right)^{n}+102400\left(1+a^{2}+8 a^{4}+5 a^{6}+a^{8}\right)^{n} \\
& +6400\left(1+2 a^{2}+5 a^{4}+4 a^{6}+4 a^{8}\right)^{n}+29696\left(1+a^{2}+7 a^{4}+3 a^{6}+4 a^{8}\right)^{n} \\
& +4710.4\left(1+10 a^{4}+5 a^{8}\right)^{n}+29184\left(1+8 a^{4}+4 a^{6}+3 a^{8}\right)^{n} \\
& +46592\left(1+7 a^{4}+6 a^{6}+2 a^{8}\right)^{n}+63488\left(\left(1+a^{2}\right)\left(1+4 a^{2}+3 a^{4}\right)\right)^{n} \\
& \left.+3942.4\left(1+5 a^{4}+10 a^{6}\right)^{n}\right]+\ldots
\end{aligned}
$$

At $a=0$, we have the correct expression obtained before, ${ }^{[17]}$

$$
\begin{aligned}
& E_{1}(g)=-g+30 g^{2}-1056 g^{3}+40176 g^{4}-1600819.2 g^{5} \\
& \quad+65774592 g^{6}-2762461769 g^{7}+1.17944875 \times 10^{11} g^{8}-\ldots
\end{aligned}
$$


The string susceptibility $\tilde{\gamma}_{\text {string }}$ is obtained by the ratio method of (5) as shown in Fig.2a and Fig.2b for $n=1$ and $n=3$ respectively. Since the successive lines in Fig. 2 are approaching smoothly to the line $\tilde{\gamma}_{\text {string }}=2$ especially for the small value of $a(a<0.25)$, the results support the result $\tilde{\gamma}_{s t r i n g}=2$. For $n=3$ there is a tiny peak around $a=0.2$. Even if this relation is violated, the value of $\tilde{\gamma}_{\text {string }}$ remains close to two. Other cases of different $n$ have similar curve as Fig.2 and the relation of (10) seem to hold at least when the critical value $a$ remains to be small enough.

For $n=2$ case, we find that the coefficient $C_{k}$ in genus one behaves as (8) without $\log k$ correction. With the assumption of the $\log k$ correction, we are unable to hold the relation $\tilde{\gamma}_{\text {string }}=2$. This rules out logarithmic terms at genus one.

We have also developed the expansion for the genus-two case up to order $g^{6}$. By the simple ratio method, in which the ratio $C_{k} / C_{k-1}$ is plotted against $1 / k$, we find the same value of the critical cosmological constant $A$ from three different genus cases. It seems that genus one case gives most reliable value of the critical cosmological constant $A$. The scaling relation of (9) seems to hold. The renormalization group approach for the matrix model has been investigated. ${ }^{[18]}$ We will discuss this approach for the present model elsewhere.

The authors thank Al.Zamolodchikov for his interest and his comments.

\section{References}

1. E. Brézin and S. Hikami, Phys. Lett. B283 (1992) 203.

2. C. Baillie and D. Johnston, Phys. Lett. B286 (1992) 44.

3. S. M. Catterall, J. B. Kogut and R. L. Renken, a preprint, hep-lat.9206021.

4. J. Ambjorn, B. Durhuus and J. Frohlich, Nucl. Phys. B257 (1985) 433.

5. F. David, Nucl. Phys. B257 (1985) 543.

6. D.V. Boulatov, V.A. Kazakov, I. Kostov and A. Migdal, Nucl. Phys. B275 (1986) 641. 
7. D.V. Boulatov and V.A. Kazakov, Phys. Lett. B214 (1988) 581.

8. E. Brézin, A. Fujita and S. Hikami, Phys. Rev. Lett. 65 (1990) 1949.

9. M. L. Mehta, Comm. Math. Phys. 49 (1981) 327.

10. E. Brézin, C. Itzykson, G. Parisi and J. B. Zuber, Comm. Math. Phys. 59 (1978) 35.

11. E. Brézin, V. Kazakov and Al. A. Zamolodchikov, Nucl. Phys. B338 (1990) 673.

P. Ginsparg and J. Zinn-Justin, Phys. Lett.B240 (1990) 333.

D. J. Gross and N. Miljkovic, Phys. Lett.B238 (1990) 217.

G. Parisi, Phys. Lett. B238 (1990) 209.

12. V.G.Knizhnik, A.M.Polyakov and A.A.Zamolodchikov, Mod. Phys.Lett.A3 (1988) 819.

F.David, Mod. Phys. Lett.A3 (1988) 207.

J. Distler and H.Kawai, Nucl. Phys. B231 (1989) 509.

13. D. Bessis, Comm. Math. Phys. 69 (1979) 147.

14. E. Brezin and J. Zinn-Justin, Phys. Letters B288 (1992) 54. 


\section{Figure caption}

Fig.1a $\gamma_{\text {string }}$ for $\mathrm{n}=1$ Ising case. At $\mathrm{a}=1 / 4, \gamma_{\text {string }}$ becomes -0.34 . The lines a,b,c and $\mathrm{d}$ are Padé analysis of (6) from order $g^{5}, g^{6}, g^{7}$ and $g^{8}$, respectively.

Fig.1b $\gamma_{\text {string }}$ for $\mathrm{n}=4(\mathrm{c}=2)$. a,b,c and $\mathrm{d}$ are same as Fig.1a.

Fig.1c $\gamma_{\text {string }}$ for $\mathrm{n}=6(\mathrm{c}=3)$. a,b,c and $\mathrm{d}$ are same as Fig.1a. The broken lines are guided lines for the extrapolation.

Fig.2a. $\tilde{\gamma}_{\text {string }}$ of $\mathrm{n}=1$ for genus one. The broken line is $\tilde{\gamma}_{\text {string }}=2$. The lines a,b,c and d are the result of the ratio method (5) for order $g^{3}, g^{4}, g^{5}$ and $g^{6}$, respectively.

Fig.2b $\tilde{\gamma}_{\text {string }}$ of $\mathrm{n}=3$ for genus one. The lines a,b,c and $\mathrm{d}$ are same as Fig.2a. 